\title{
Convection induced by instabilities in the presence of a transverse seepage
}

\author{
Takeshi Akinaga ${ }^{1,3}$, Tomoaki Itano ${ }^{2}$ and Sotos Generalis ${ }^{3}$ \\ ${ }^{1}$ Marie Sklodowska Curie International Incoming Fellow \\ ${ }^{2}$ Department of Pure and Applied Physics, \\ Faculty of Engineering Science, \\ Kansai University, Osaka 564-8680, Japan and \\ ${ }^{3}$ System Analytics Research Institute, Aston University, \\ Birmingham, B4 YET, United Kingdom*
}

(Dated: December 28, 2015)

\begin{abstract}
The transition of laterally heated flows in a vertical layer and in the presence of a streamwise pressure gradient is examined numerically for the case of different values Prandtl number. The stability analysis of the basic flow for the pure hydrodynamic case $(\operatorname{Pr}=0)$ was reported in [1]. We find that in the absence of transverse pumping the previously known critical parameters are recovered [2], while as the strength of the Poiseuille flow component is increased the convective motion is delayed considerably. Following the linear stability analysis for the vertical channel flow our attention is focused on a study of the finite amplitude secondary travelling-wave (TW) solutions that develop from the perturbations of the transverse roll type imposed on the basic flow and temperature profiles. The linear stability of the secondary TWs against three-dimensional perturbations is also examined and it is shown that the bifurcating tertiary flows are phase-locked to the secondary TWs.
\end{abstract}




\section{INTRODUCTION AND MOTIVATION}

Theoretical investigations aided by the advance of powerful hardware and parallel experimental studies of the stability of plane parallel shear flows have provided significant insights in identifying the mechanisms of instability and transition from the laminar to the turbulent state of shear fluid flow, via the approach based on the sequence of bifurcations. Parallel shear flows include the well studied plane Couette flow, plane Poiseuille flow (PPF), homogeneously heated flow, laterally heated flow (LHF) and Rayleigh-Bénard (natural) convection. In [2] the parallel shear flow LHF was studied for the case of $P r=0$ without the imposition of a constant pressure gradient was presented. In [1] the linear stability for the $\operatorname{Pr}=0$ case was examined for a wide range of the Grashof and Reynolds numbers in order to extract only the fluid dynamic instability mechanisms. The present work complements and extends the work of [1] by examining the nonlinear flow that emerges at the stability boundary of the basic flow configuration. The general form of the Navier-Stokes equations can be expressed by $\frac{\partial \boldsymbol{\Phi}}{\partial t}=\boldsymbol{M}(\boldsymbol{R}) \boldsymbol{\Phi}+\boldsymbol{N}(\boldsymbol{\Phi}, \boldsymbol{\Phi})$. In this set of coupled partial differential equations, $\boldsymbol{\Phi}$ describes the state of the system, $\boldsymbol{M}(\boldsymbol{R})$ is a linear and $\boldsymbol{N}(\cdot, \cdot)$ is a nonlinear operator that involve partial derivatives. $\boldsymbol{R}$ represents collectively the parameters of the system, such as the angle of inclination of the channel flow, the Prandtl, the Rayleigh (or Grashof), the Reynolds and the wave numbers. In our sequential bifurcation approach we first obtain the stability of the basic state $\boldsymbol{\Phi}_{0}$, which satisfies:

$$
0=\boldsymbol{M}(\boldsymbol{R}) \Phi_{0}+\boldsymbol{N}\left(\boldsymbol{\Phi}_{0}, \boldsymbol{\Phi}_{0}\right)
$$

via the introduction of infinitesimal disturbances, $\widetilde{\boldsymbol{\Phi}}$ and by ignoring the nonlinear terms:

$$
\frac{\partial \widetilde{\boldsymbol{\Phi}}_{0}}{\partial t}=\boldsymbol{M}(\boldsymbol{R}) \widetilde{\boldsymbol{\Phi}}_{0}+\boldsymbol{N}\left(\boldsymbol{\Phi}_{0}, \widetilde{\boldsymbol{\Phi}}_{0}\right)+\boldsymbol{N}\left(\widetilde{\boldsymbol{\Phi}}_{0}, \boldsymbol{\Phi}_{0}\right) .
$$

The nonlinear states grow from the stability boundary of the basic state, that is typically characterised by $f_{0}(\boldsymbol{R})=0$, where $f_{0}$ is the neutral surface of the 
basic state in parameter space $\boldsymbol{R}$. If we repeat the aforementioned procedure of perturbation on the obtained nonlinear states, we could find a $f_{i}(\boldsymbol{R})=0$, where $f_{i}, i=(1,2, \ldots)$, is the corresponding neutral surface of the nonlinear state, thus defining a successive sequence of bifurcations for the higher order states en route to turbulence.

Motivated by the desire to understand the transition from laminar flow to the turbulent state for this (mixed convection) problem, we revisited the pure hydrodynamic case, first studied in [1] through to tertiary level, and also extended it to the case $\operatorname{Pr}=0.71$. In the following section we formulate the problem and in section 3 we show the numerical method that we employ to investigate the linear stability of our basic steady state. In section 4 we study the nonlinear states that are generated at the stability boundary of the basic state of our problem. In section 5 we study the stability of the bifurcated secondary flow and we therefore found the manifold of points where the tertiary states are generated. In section 6 we provide our conclusions and we identify avenues for future research.

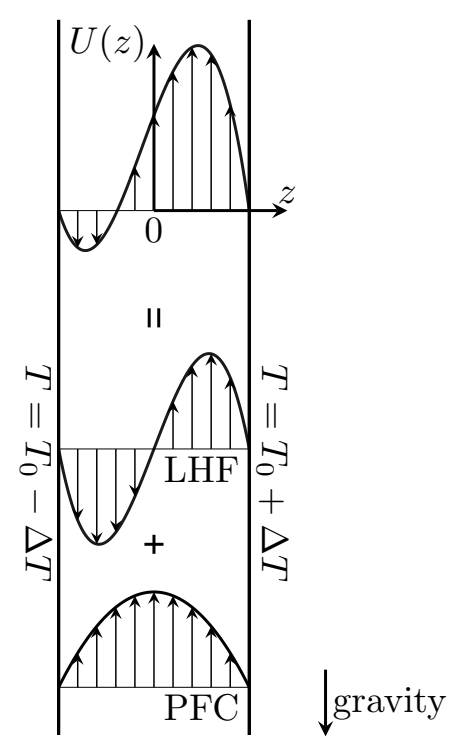

FIG. 1: Geometrical configuration of the problem. In this figure $-d \leq z \leq d$. 


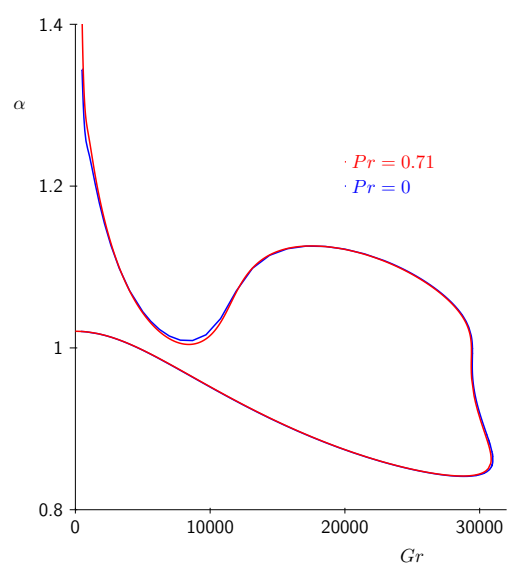

(a)

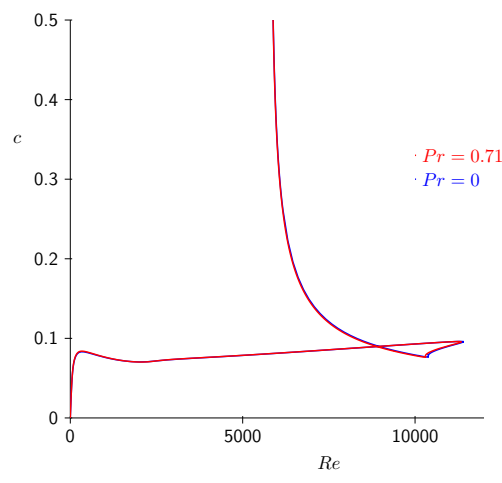

(c)

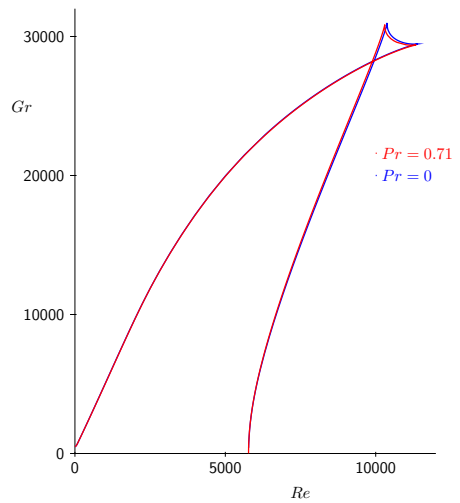

(e)

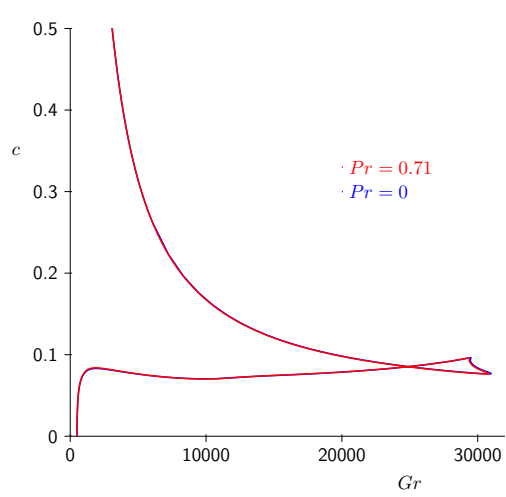

(b)

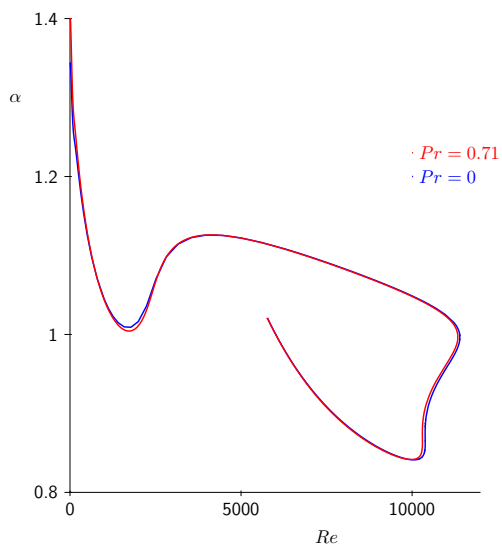

(d)

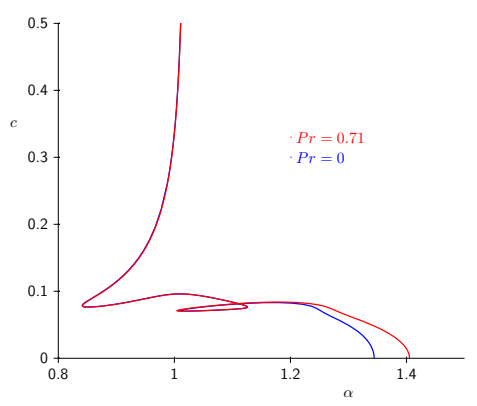

(f)

FIG. 2: (a)-(f): The curves for the critical conditions $\left(\alpha_{c}, R e_{c}, G r_{c}, c_{c}\right)$, i.e. the local minimum/maximum points on the neutral curves in the $(\alpha, G r, R e)$ space, for the cases $\operatorname{Pr}=0$ (darker shade), $\operatorname{Pr}=0.71$. The two curves are almost indistinguishable. For $\operatorname{Pr}_{4}=0$ and (d-e)) see also [1]. 


\section{MATHEMATICAL FORMULATION AND PRIMARY STABILITY}

We consider an incompressible Boussinesq Newtonian fluid bounded by two vertical parallel plates of infinite extent with different temperatures $T_{0}+\Delta T$ and $T_{0}-\Delta T$, subject to a pressure gradient deviation from the hydrostatic pressure, see Fig. 1. The origin of the Cartesian coordinates system is positioned in the midplane of the fluid layer of width $2 d$, taking $x, y, z$ coordinates as the streamwise, spanwise and wall normal directions with unit vectors $\boldsymbol{i}, \boldsymbol{j}, \boldsymbol{k}$. We assume here the state satisfies the periodic boundary conditions with wave numbers $\alpha$ and $\beta$ for the $x$ and $y$ directions, respectively. For the non-dimensional form of the velocity vector, the temperature and pressure deviations from the basic state as $\boldsymbol{u}, \theta$ and $\pi$, we obtain the non-dimensional form of the equations of motion in the following form:

$$
\begin{aligned}
\frac{\partial}{\partial t} \boldsymbol{u}+\boldsymbol{u} \cdot \nabla \boldsymbol{u} & =-\nabla \Pi+\theta \sin \chi \boldsymbol{i}+\nabla^{2} \boldsymbol{u} \\
\frac{\partial}{\partial t} \theta+\boldsymbol{u} \cdot \nabla \theta & =\operatorname{Pr}^{-1} \nabla^{2} \theta \\
\boldsymbol{\nabla} \cdot \boldsymbol{u} & =0 .
\end{aligned}
$$

The Boussinesq approximation has been used in that the material properties are assumed to be constant except for the linear temperature dependence of the density, which has been taken into account in the buyoancy term only. The angle of inclination of the channel flow is indicated by $\chi$. Only the vertical case $\left(\chi=90^{\circ}\right)$ will be addressed in this manuscript. The physical properties of the system are characterised by the three non-dimensional parameters $G r=g \gamma \Delta T d^{3} / \nu^{2}$, the Grashof number that gives the strength of the heating, $R=U_{\max } d / \nu$, the Reynolds number that measures the strength of the applied pressure gradient in the streamwise direction $\left(U_{\max }\right.$ is the speed of Poiseuille laminar flow at the origin of the coordinate system) and the Prandtl number $\operatorname{Pr}=\nu / \kappa$. Here $\kappa$ is the thermal diffusivity, $\nu$ is the kinematic viscosity, $\gamma$ is the coefficient of thermal 
TABLE I: Critical conditions for $\operatorname{Pr}=0$, and $L=49$ (in brackets the relative difference between the two truncation levels $L=49$ and 64 , is also given). The results for $\operatorname{Pr}=0.71$ are marginally different, as can be seen from Fig. 2 .

\begin{tabular}{cccc}
\hline$R e_{\mathrm{c}}$ & $\alpha_{\mathrm{c}}$ & $G r_{\mathrm{c}}$ & $c_{\mathrm{c}}$ \\
\hline 0.0001 & $1.34414712\left(<10^{-10}\right)$ & $4.95628445 \times 10^{2}\left(<10^{-10}\right)$ & $5.81063092 \times 10^{-5}\left(<10^{-10}\right)$ \\
0.001 & $1.34414712\left(<10^{-10}\right)$ & $4.95628445 \times 10^{2}\left(<10^{-10}\right)$ & $5.81063092 \times 10^{-4}\left(<10^{-10}\right)$ \\
0.01 & $1.34414712\left(<10^{-10}\right)$ & $4.95628449 \times 10^{2}\left(<10^{-10}\right)$ & $5.81063092 \times 10^{-3}\left(<10^{-10}\right)$ \\
0.1 & $1.34414690\left(<10^{-10}\right)$ & $4.95628824 \times 10^{2}\left(<10^{-10}\right)$ & $5.81063031 \times 10^{-2}\left(<10^{-10}\right)$ \\
1 & $1.34412514\left(<10^{-10}\right)$ & $4.95666335 \times 10^{2}\left(<10^{-10}\right)$ & $5.81056886 \times 10^{-1}\left(<10^{-10}\right)$ \\
200 & $1.22172873\left(<10^{-10}\right)$ & $1.24286270 \times 10^{3}\left(<10^{-10}\right)$ & $1.00083364 \times 10^{2}\left(<10^{-10}\right)$ \\
400 & $1.15309918\left(<10^{-10}\right)$ & $2.16843720 \times 10^{3}\left(<10^{-10}\right)$ & $1.79966790 \times 10^{2}\left(<10^{-10}\right)$ \\
600 & $1.10463107\left(<10^{-10}\right)$ & $3.10599624 \times 10^{3}\left(<10^{-10}\right)$ & $2.51355252 \times 10^{2}\left(<10^{-10}\right)$ \\
800 & $1.06988524\left(<10^{-10}\right)$ & $4.05190483 \times 10^{3}\left(<10^{-10}\right)$ & $3.17795977 \times 10^{2}\left(<10^{-10}\right)$ \\
1000 & $1.04472297\left(<10^{-10}\right)$ & $5.00361256 \times 10^{3}\left(<10^{-10}\right)$ & $3.81045816 \times 10^{2}\left(<10^{-10}\right)$ \\
2000 & $1.01645695\left(<10^{-10}\right)$ & $9.71432784 \times 10^{3}\left(<10^{-10}\right)$ & $6.83103956 \times 10^{2}\left(<10^{-10}\right)$ \\
3000 & $1.10836575\left(<10^{-10}\right)$ & $1.38251742 \times 10^{4}\left(<10^{-10}\right)$ & $1.01675867 \times 10^{3}\left(<10^{-10}\right)$ \\
4000 & $1.12588160\left(<10^{-10}\right)$ & $1.71850746 \times 10^{4}\left(<10^{-10}\right)$ & $1.30729842 \times 10^{3}\left(<10^{-10}\right)$ \\
5000 & $1.12217952\left(8.91 \times 10^{-9}\right)$ & $1.99664136 \times 10^{4}\left(5.01 \times 10^{-9}\right)$ & $1.56885650 \times 10^{3}\left(6.37 \times 10^{-9}\right)$ \\
6000 & $1.11184398\left(8.99 \times 10^{-9}\right)$ & $2.22730160 \times 10^{4}\left(4.49 \times 10^{-9}\right)$ & $1.81032286 \times 10^{3}\left(1.10 \times 10^{-8}\right)$ \\
7000 & $1.09851537\left(1.27 \times 10^{-7}\right)$ & $2.41976883 \times 10^{4}\left(1.07 \times 10^{-7}\right)$ & $2.03571153 \times 10^{3}\left(6.88 \times 10^{-8}\right)$ \\
8000 & $1.08351866\left(6.46 \times 10^{-8}\right)$ & $2.58169954 \times 10^{4}\left(2.71 \times 10^{-8}\right)$ & $2.24735408 \times 10^{3}\left(2.67 \times 10^{-8}\right)$ \\
9000 & $1.06717744\left(1.50 \times 10^{-7}\right)$ & $2.71848163 \times 10^{4}\left(2.21 \times 10^{-7}\right)$ & $2.44624385 \times 10^{3}\left(4.09 \times 10^{-8}\right)$ \\
10000 & $1.04881385\left(4.48 \times 10^{-7}\right)$ & $2.83272734 \times 10^{4}\left(9.00 \times 10^{-7}\right)$ & $2.63135029 \times 10^{3}\left(1.82 \times 10^{-7}\right)$ \\
\hline & & &
\end{tabular}


expansion and $g$ is the acceleration due to gravity. For the non-dimensional description of the problem we employ $d, d^{2} / \nu$ and $\Delta T / \operatorname{Pr} G r$, as the units of length, time and temperature, respectively. Eqs.(2-4) are supplemented by the boundary conditions:

$$
\boldsymbol{u}=\theta=0 \text { at } z= \pm 1
$$

The basic flows are provided by eqs.(2)-(3) and are given by:

$$
U_{0}(z)=-\frac{G r}{6}\left(z^{3}-z\right)+\operatorname{Re}\left(1-z^{2}\right), T_{0}(z)=G r z \quad \text { for } \quad|z| \leq 1 .
$$

In order to be able to identify other, than the laminar, solutions for eqs. (2-4) it is convenient to introduce a general representation for solenoidal vector fields [3] and to write:

$$
\boldsymbol{u}=\check{U} \boldsymbol{i}+\nabla \times(\nabla \times \phi \boldsymbol{k})+\nabla \times \psi \boldsymbol{k}
$$

where the poloidal and toroidal potentials, $\phi, \psi$, respectively, are uniquely defined if their averages over the planes $z=$ constant vanishes. We introduce $\widehat{U}(z)=$ $U_{0}(z)+\check{U}(z)$ and $\widehat{T}(z)=T_{0}(z)+\check{T}(z)$ and this enables us to write for the mean flow and mean temperature $\check{U}, \check{T}$ respectively:

$$
\begin{aligned}
\partial_{z}^{2} \check{U}+\check{T}+\partial_{z} \overline{\Delta_{2} \phi\left(\partial_{x} \partial_{z} \phi+\partial_{y} \psi\right)} & =\partial_{t} \check{U} \\
\partial_{z}^{2} \check{T}+\operatorname{Pr}_{z} \overline{\Delta_{2} \phi \theta} & =\operatorname{Pr}_{t} \check{T}
\end{aligned}
$$

where the overbar denotes an average over the planes $z=\operatorname{constant}(s)$, and $\Delta_{2}$ denotes the two dimensional Laplacian, $\Delta_{2}=\partial^{2} / \partial x^{2}+\partial^{2} / \partial y^{2}$. Since we anticipate a vanishing mean flow in the $y$-direction, we have neglected it in expression (7). Equations (8-9) have been obtained by taking the $x, y$-average of eqs.(2-3) and by subtracting the basic solution $U_{0} \boldsymbol{i}$. In order to obtain transverse vortices we use the Fourier expansions for the variables $(\phi, \psi, \theta)$ in $\boldsymbol{\Phi}=(\boldsymbol{u}, \theta)=(u, v, w, \theta)$, see also $[2,6-8]$ : 


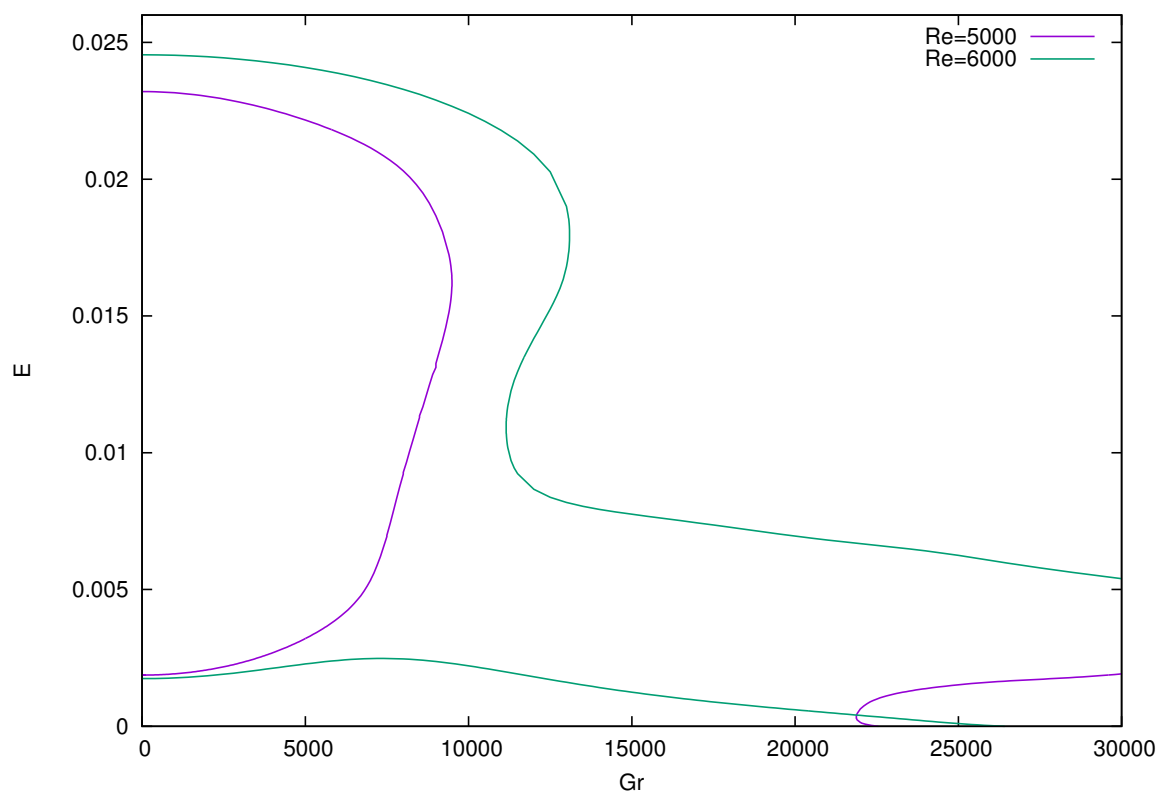

FIG. 3: Total energy E (see [4], [5]) for different (two-dimensional) states are indicated for fixed $\operatorname{Pr}=0.71, R e=5,000$ (darker shade), $R e=6,000$ and a variety of $G r$ values. Here $\alpha=1.324$.

$$
\begin{aligned}
\phi(x, y, z, t) & =\sum_{n=0}^{N} \sum_{\substack{m=-M \\
(m, l) \neq(0,0)}}^{M} \sum_{l=-L}^{L} a_{n m l}\left(1-z^{2}\right)^{2} T_{n}(z) \exp (\mathrm{i} m \alpha(x-c t)+\mathrm{i} l \beta y), \\
\theta(x, z, t) & =\sum_{n=0}^{N} \sum_{\substack{m=-M \\
(m, l) \neq(0,0)}}^{M} \sum_{\substack{l=-L \\
L}}^{L} b_{n m l}\left(1-z^{2}\right) T_{n}(z) \exp (\mathrm{i} m \alpha(x-c t)+\mathrm{i} l \beta y),
\end{aligned}
$$

while we write:

$$
\check{U}=\sum_{n=0}^{N} C_{n}\left(1-z^{2}\right) T_{n}(z), \check{T}=\sum_{n=0}^{N} D_{n}\left(1-z^{2}\right) T_{n}(z),
$$

where $N$ and $M$ are the truncation levels for the complex coefficients $a_{n m}$, and $T_{n}(z)$ is the $n$-th order Chebyschev polynomial. We have incorporated the phase 


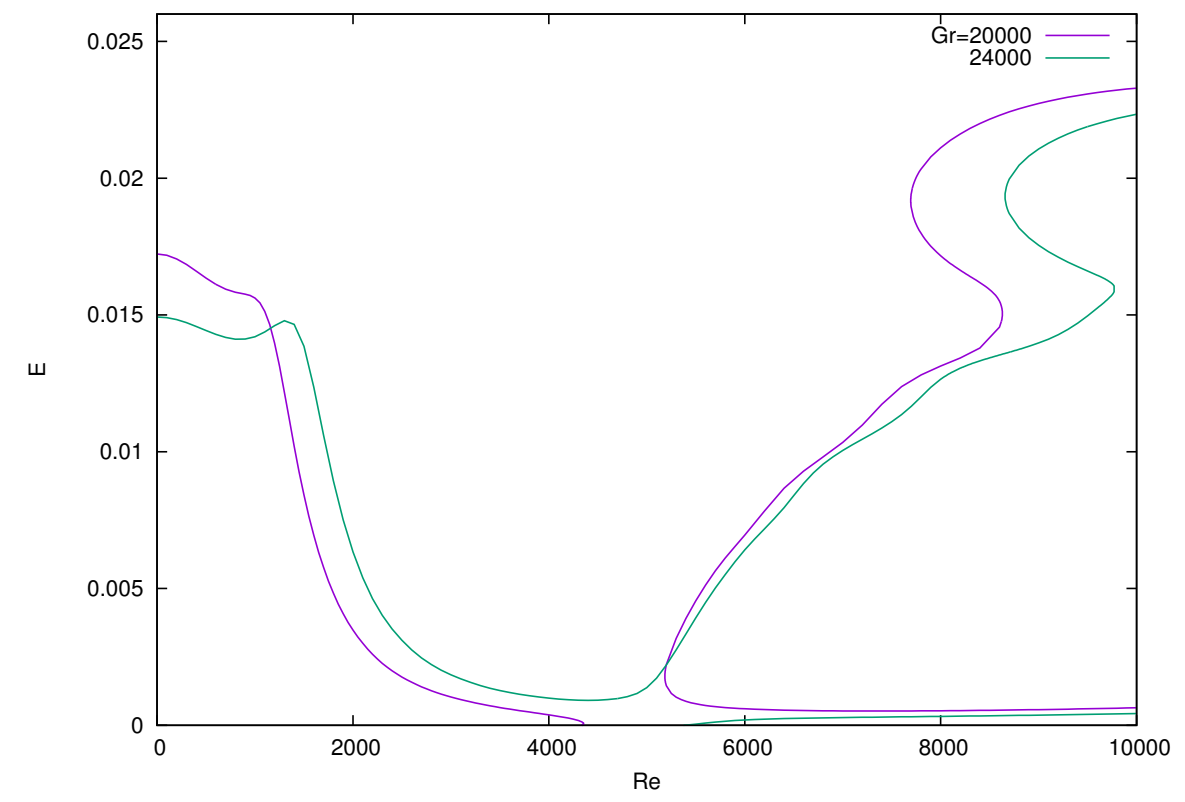

FIG. 4: Total energy E (see [4], [5]) for different (two-dimensional) states are indicated for fixed $\operatorname{Pr}=0.71, G r=20,000$ (darker shade), $G r=24,000$ and a variety of $R e$ values. Here $\alpha=1.324$.

velocity $c$ in the expansion, so that calculations can be performed on a moving frame that is phase locked with the nonlinear solutions bifurcating from the neutral curve boundaries. The factor $\left(1-z^{2}\right)^{2}$ has been inserted in the expression for $\psi$ in order to take into account the boundary conditions expressed by eqs. (5). By substituting the expansions (10) into the streamwise projection of the curl and double curl of (2), rewriting (3) using (7), and finally multiplying the resulting equations by $\int_{0}^{2 \pi / \alpha} d x \exp \mathrm{i} l \alpha x, l=1,2, \ldots$, we can evaluate the expansions (10) at each collocation point, i.e. we obtain a set of nonlinear algebraic equations for the complex coefficients $a_{n m}$. Further details of the numerical method employed here to obtain nonlinear solutions have been presented recently in $[6]$ and $[7,8]$ in relation to different types of shear flows. We elaborate on the results of our simulations in the next section. For the numerical solution of the resulting systems 


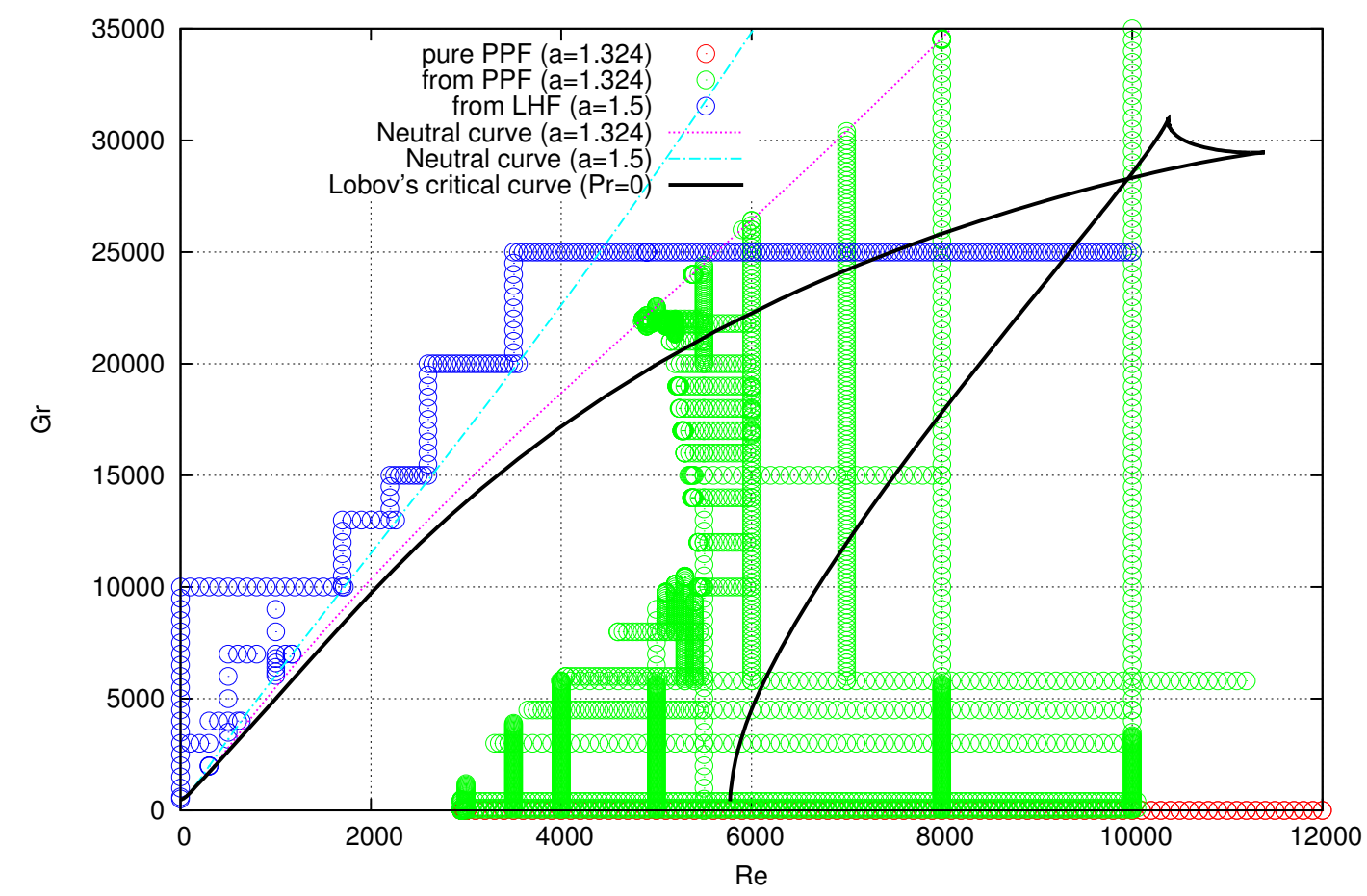

FIG. 5: A variety of two-dimensional states indicated by open circles for the case of $\operatorname{Pr}=0.71$. Light shaded circles for $3,000 \leq R e \leq 12,000$ are for $\alpha=1.324$ and PPF, while darker shaded curves are for $\alpha=1.5$ and LHF, respectively. There exists a variety of combinations for $G r, R e$ for which the two-dimensional states exist within the stable region of the basic state, as low as $R e \approx 3,000$. Neutral curves for fixed $\alpha=1.324$ and $\alpha=1.5$ are also depicted, as indicated.

of equations a truncation scheme must be introduced. The boundaries of Fig (2) and the numerical values of Table I are obtained by setting $m=1, l=0$ in eq.(10) and by ignoring the nonlinear effects of the mean flow and temperature deviations given by eqs.( 8-9). The truncation level is also indicated. The introduction of a Poiseuille flow delays the (primary) instability for thermal convection in a vertical slot. The ordinate for $R e=0$ is the critical condition for pure LHF, see [1], [2]. 


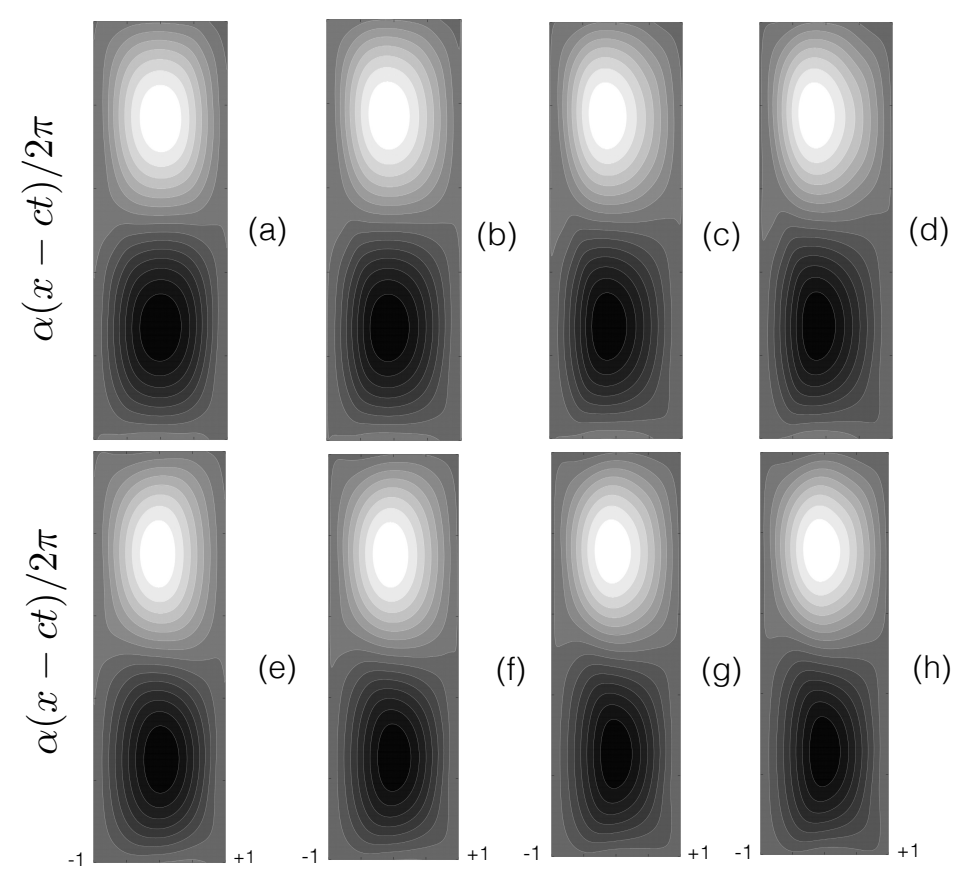

FIG. 6: Two dimensional contour plots for the stream function $\psi=\partial_{x} \phi$ for $\operatorname{Pr}=0.71$ and for (a)-(d): $\alpha=1.02, G r=0,10,000,20,000,25,000,(\mathrm{e})-(\mathrm{h})$ : $\alpha=1.324, G r=0,10,000,20,000,25,000$. All contour plots are presented for $R e=6,000$.

As can be seen from Figs (2) the critical curve for the case of $\operatorname{Pr}=0.71$ is marginally different from the case of a high thermal conductivity liquid (i.e. $\operatorname{Pr}=0)$. In this case the dissipation of the thermal disturbances and the case can be treated as isothermal [1]. In the absence of a Poiseuille flow component (PFC), see Fig 2 , the critical condition is $\left(G r_{c}, \alpha_{c}\right)=(495.63,1.344)$ [2]. In Table I we provide detailed information about the critical condition dependence on the truncation level that is employed here. It is evident from Fig 2 and Table I, that the introduction of a PFC stabilises considerably the convective motion, so for example for $R e=10,000, G r_{c}>28,000$. For the case $G r=0$ we obtain the known PPF critical values. In Fig.2 we show the dependance of wavenumber for 
which we attain an extremum depending on the values of Re. We see that the function $\alpha(R e)$ is a smooth function up to the case when the bi-critical point is reached. The upper part of the curve $\alpha(R e)$ corresponds to the upper section of the (critical conditions) stability boundary and the lower part of the curve corresponds to the lower section of the ability boundary. Various other dependences are shown, including the dependence of the phase velocity $c$ of the disturbances on the value of $R e, G r$. Finally we note that the bi-critical point coordinates for the case of $\operatorname{Pr}=0.71$ are: $(R e=10,000, G r=28,000)$.

\section{SECONDARY STATES}

We now commence a brief analysis of our results. In Fig. 3 we show the total energy (see [4], [5]) of the two-dimensional (two-dimensional) solutions for fixed $R e$ and variable $G r$. In Fig.3 the left red curve depicts the nonlinear state in pure Poiseuille flow (PPF) for $\alpha=1.324$ (see also Fig.(5)) at $R e=5,000$ and has been continued for a range of values of $G r$. We depict both (upper and lower) branches of this solution in this figure This particular solution is within the stable region (for the basic state) and never attains the solution with $G r=10,000$ (see also Fig.(5) for comparison). The second red curve represents the state that bifurcates subcritically at $G r \approx 22,500$ and can be extended to join the pure PPF region. As the value of Reynolds number increases the two red curves join and for $R e=6,000$ there are two topologically different curves that are depicted in blue. The upper blue curve does not originate either from the PPF or pure LHF regions, and can be continued for a wide range of $G r$. The lower blue curve depicts the subcritical bifurcation of Fig.(5) at $G r \approx 26,420$ and extends to the PPF region $(R e=0)$.

In Fig.4 we show the total energy (see [4], [5]) of the two-dimensional solutions for fixed $G r$ and variable Re. In this Fig.4 the left red curve (for $G r=20,000$ ) depicts the nonlinear state that is generated by a supercritical bifurcation (see 

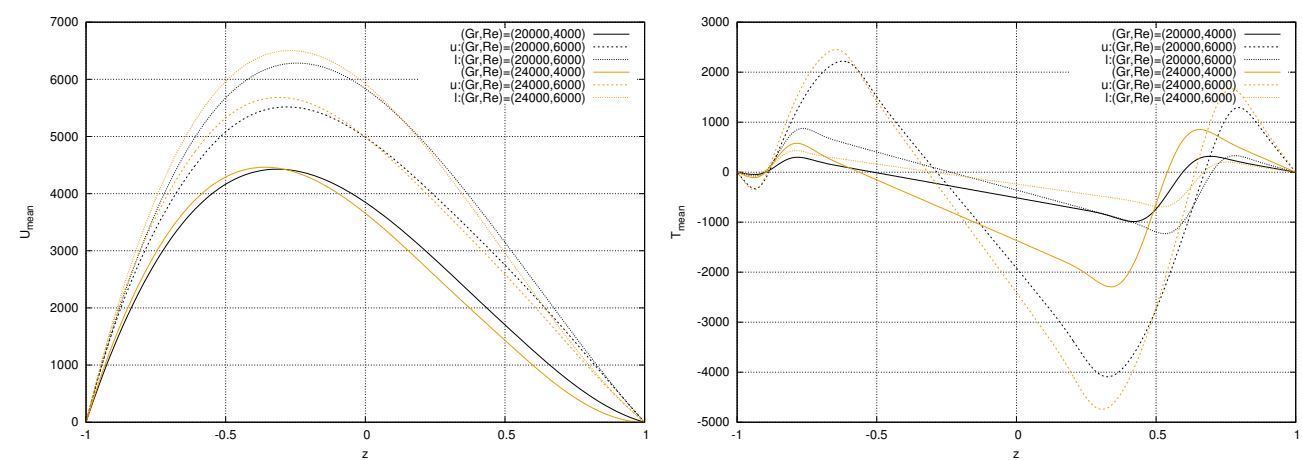

FIG. 7: The total mean flow and mean temperature profiles,

$U_{\text {mean }}=\widehat{U}(z)=U_{0}(z)+\check{U}(z)$ and $T_{\text {mean }}=\widehat{T}(z)=T_{0}(z)+\check{T}(z)$ respectively, as functions of $\mathrm{z}$ for different Grashof and Reynolds numbers as indicated. The wavenumber $\alpha$ of transverse vortices is fixed at 1.324. For selected values of $G r, R e$ both the upper (indicated as $u$ ) and the lower branch (indicated as $l$ ) profiles are depicted.

also the red neutral curve of Fig.(5) for $\alpha=1.324$ ) at $R e \approx 4,200$. This state is depicted until the pure LHF is reached $(R e=0)$. The solution for vanishing $R e$, is outside the stable region of the two-dimensional states in the pure LHF region of [9] and is therefore unstable. In Fig.4 the right red curve depicts the nonlinear state that has been continued for a range of values of $R e$. We depict both (upper and lower) branches of this solution in this figure and the turning point of the solutions is for $R e \approx 5,200$. Both beaches of this particular solution extend within the stable region (for the basic state) (see also Fig.(5) for comparison). The blue curve on the right hand side represents the state that bifurcates supercritically at $G r \approx 22,500$ and can be extended to exist within the neutrally stable region. As the value of the Gr number has increased the two red curves for $G r=20,000$ (the left hand side red curve and the upper branch of the right hand side red curve) join to form one blue curve that extends over a wide spectrum of values of $R e$. The upper blue curve does not originate either from the PPF or pure LHF regions. The 
lower blue curve depicts the supercritical bifurcation of Fig.(5) at $R e \approx 5,500$ and extends to the neutrally stable PPF region $(R e=0)$.

\section{Some comments for fixed $\alpha$ - choice of $\alpha$}

The secondary states for $R e \geq 3000, G r \geq 22,000$ and for $\alpha=1.324$ are subcritical. This value of the wavenumber was chosen so that we can find the smallest possible value of $R e$ that can be achieved for our secondary states. For $\alpha=1.02$ we can find a value of $R e=4,500$ that can be reached. Continuing from $\alpha=1.02$ to $\alpha=1.324$ we can find that the secondary (subcritical state) can reach values of $R e=2,500$. This does not preclude the possibility that for a different $\alpha$ we can reach even lower values of $R e$. Note that experimentally values of $R e=1,500$ have been recorded. Of course it could be reached from our 3D state - see later on. There is no connection of the secondary states in blue and green, as they refer to solutions with different wavenumbers. In Figure 7 we have plotted the profiles of the mean flow and mean temperature for various values of the Grashof and Reynolds numbers. We have also depicted profiles for both the upper and lower branches. For $R e=4000$ and $R e=6000$ there are single curves plotted to show the effect of the imposed pressure gradient. Figure 7 shows that the profile of the mean flow does not retain its cubic shape. A saturation effect is noticeable in so far as there is relatively little change between the curves for upper and lower branches, but the effect of the pressure gradient is noticeable, with the higher Reynolds numbers exhibiting the largest magnitude.

\section{Some comments for convective mode domination - choice of $\alpha$}

In Fig 5 we can see that the secondary TWs penetrate into the stable region defined by the critical curves for $\operatorname{Pr}=0.71$. This is the case for the secondary states with $G r \geq 22,000$ and $\alpha=1.5$. This can be attributed to the fact that 
the LHF mode is dominant, with respect to the purely hydrodynamic mode, for these values of the Grashof number. In fact for this value of the wavenumber the nonlinear states are subcritical and it is the upper branch of the solutions that penetrate the Re stable region. Despite the fact that there exist nonlinear solutions within the linearly stable region there is an area (defined by criticality (for $G r, \alpha, R e)$ remaining that there is no presence of the secondary states, see Figure 5. The secondary states for $R e \geq 3000$ are subcritical, but the subcriticality is not strong enough to penetrate the regions for $G r<20,000$. For $\alpha<1.5$ PPF originating secondary states do not connect with the neutral curves on the convective side, see Figure 5. As mentioned above the presence of higher order states (within the bifurcation sequence of the flow) might lessen the area defined by the linear stability boundaries.

\section{INSTABILITIES OF THE SECONDARY FLOW}

We now study the stability of the secondary solutions obtained in the previous section in order to identify the bifurcation points of the tertiary states. In order to study the stability of the transverse traveling waves we superimpose three dimensional infinitesimal disturbances on the secondary equilibrium states obtained in the previous section and we seek to numerically evaluate their corresponding growth rates. As in the case of the linear stability analysis of the basic flow, growing disturbances will indicate an unstable solution. The stability or instability of the disturbance will be dictated by the real part $\sigma_{r}$ of the most dangerous eigenvalue(s).

\section{Numerical method - Eckhaus Instability}

We denote the complex infinitesimal disturbance on the nonlinear solution of the previous section, $\boldsymbol{\Phi}=(u, v, w, \theta)$, by $\widetilde{\boldsymbol{\Phi}}=(\tilde{u}, \tilde{v}, \tilde{w}, \tilde{\theta})$, respectively and we set 
for the disturbance $\widetilde{\boldsymbol{\Phi}}$ (see also [8]):

$$
\begin{aligned}
\tilde{\phi}= & \sum_{m=-\infty}^{\infty} \sum_{n=0}^{\infty} \exp \{i m a(x-c t)+i d x+i b y+\sigma t\} \\
& \times\left(1-z^{2}\right)^{2} \tilde{a}_{m n} T_{n}(z), \\
\tilde{\psi}= & \sum_{m=-\infty}^{\infty} \sum_{n=0}^{\infty} \exp \{i m a(x-c t)+i d x+i b y+\sigma t\} \\
& \left(1-z^{2}\right) \tilde{b}_{m n} T_{n}(z), \\
\tilde{\theta}= & \sum_{m=-\infty}^{\infty} \sum_{n=0}^{\infty} \exp \{i m a(x-c t)+i d x+i b y+\sigma t\} \\
& \left(1-z^{2}\right) \tilde{c}_{m n} T_{n}(z),
\end{aligned}
$$

with $T_{n}$ the $n$-th order Chebyschev polynomial, as before. The variable $\tilde{\psi}$ has been expanded in the same form as the variable $\tilde{\theta}$, because it satisfies the same boundary conditions:

$$
\tilde{\phi}=\frac{\partial \tilde{\phi}}{\partial z}=\tilde{\psi}=\tilde{\theta}=0 \quad \text { at } \quad z= \pm 1 .
$$

In this work and in order to identify the stable region of the secondary solutions, we therefore solve the generalised eigenvalue problem:

$$
Q_{i j} \tilde{x}_{j}=\sigma R_{i j} \tilde{x}_{j}
$$

in the unknown complex variables $\left\{\tilde{a}_{m n}, \tilde{b}_{m n}, \tilde{c}_{m n}\right\}$, represented by $\tilde{x}_{i}$. The matrices $Q_{i j}, R_{i j}$ are functions of the Floquet multipliers $d, b$, the wavenumber $a$, the parameters $G r, \operatorname{Pr}$ and the amplitudes of the steady state solution $\left\{a_{m n}, b_{m n}, c, C_{n}, D_{n}\right\}$. We then proceed to evaluate the real part $\sigma_{r}$ of the most dangerous eigenvalue(s) (with instability presenting itself if $\sigma_{r}>0$ ). As in the case of steady solutions the infinite system of equations (16) must be truncated for the numerical evaluation of the corresponding eigenvalues. We have used here the same truncation level as that for the identification of the two-dimensional solutions of the previous section. We note that because the disturbances $\tilde{\phi}, \tilde{\psi}, \tilde{\theta}$ are infinitesimal all terms that are of non-linear nature in (1) have been neglected. 

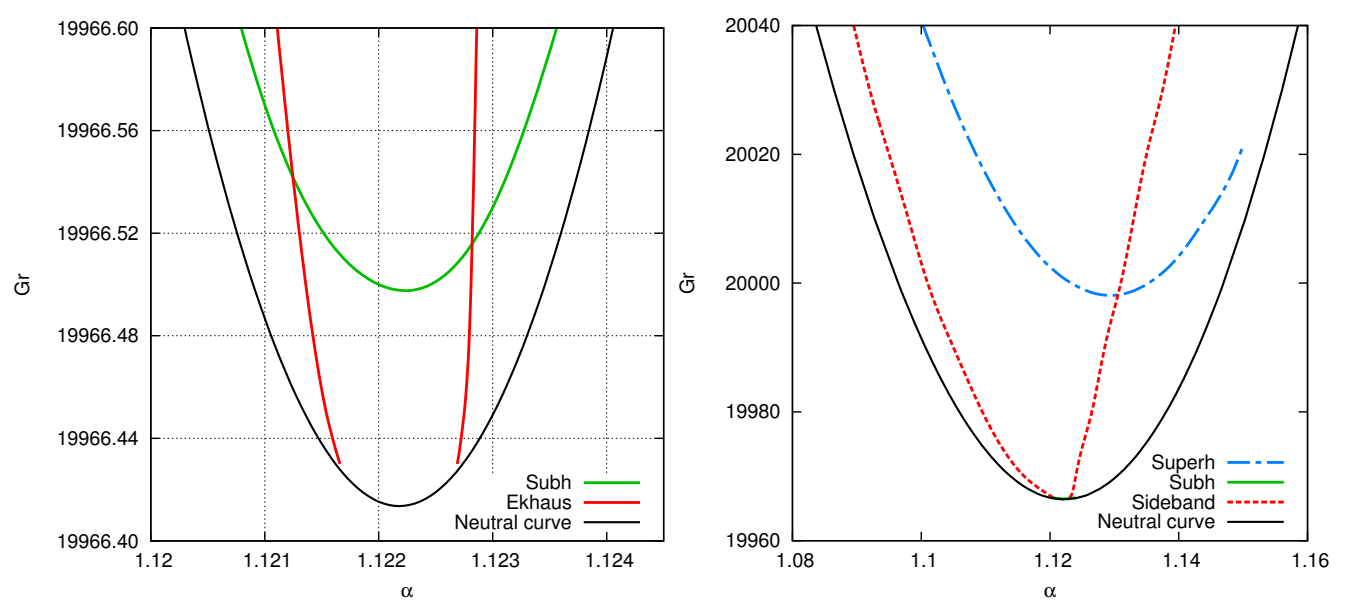

FIG. 8: Eckhaus stability boundary of two-dimensional nonlinear states for the case $\operatorname{Pr}=0$. The value of the Floquet parameter $d$ employed is as indicated.

The Floquet parameter is set to $b=0$.

As the value of $d^{2}+b^{2}$ will be assumed to be different from zero for the rest of the analysis, there is no contributions to the mean temperature and flow. Disturbances with $d=b=0$ are actually included in the analysis of the steady solution. We have not encountered the resonant boundary of [5], beyond which steady solutions could not be obtained. This boundary, for example, is due to this instability; disturbances with twice the wavenumber replace the steady solution beyond that boundary. The boundary due to resonance is not called a stability boundary, however, since disturbances of the same character (i.e. with $b=0$ ), but with finite $d$ grow more strongly than those with $d=0$ and the appropriate stability boundary is thus given by the Eckhaus curve in Figure 8. In Figure 8 we show the stability region of the transverse traveling waves in the moving frame and in the $\{G r, a\}$ parameter space for $R e=5,000$. The Eckhaus instability curve, [10], bounds the transverse (drafting) vortices towards smaller and larger wavenumbers and is characterized by $b=0$. Therefore, for any given Grashof and Prandtl numbers the Eckhaus instability curve lies within the linear stability curve. In all 


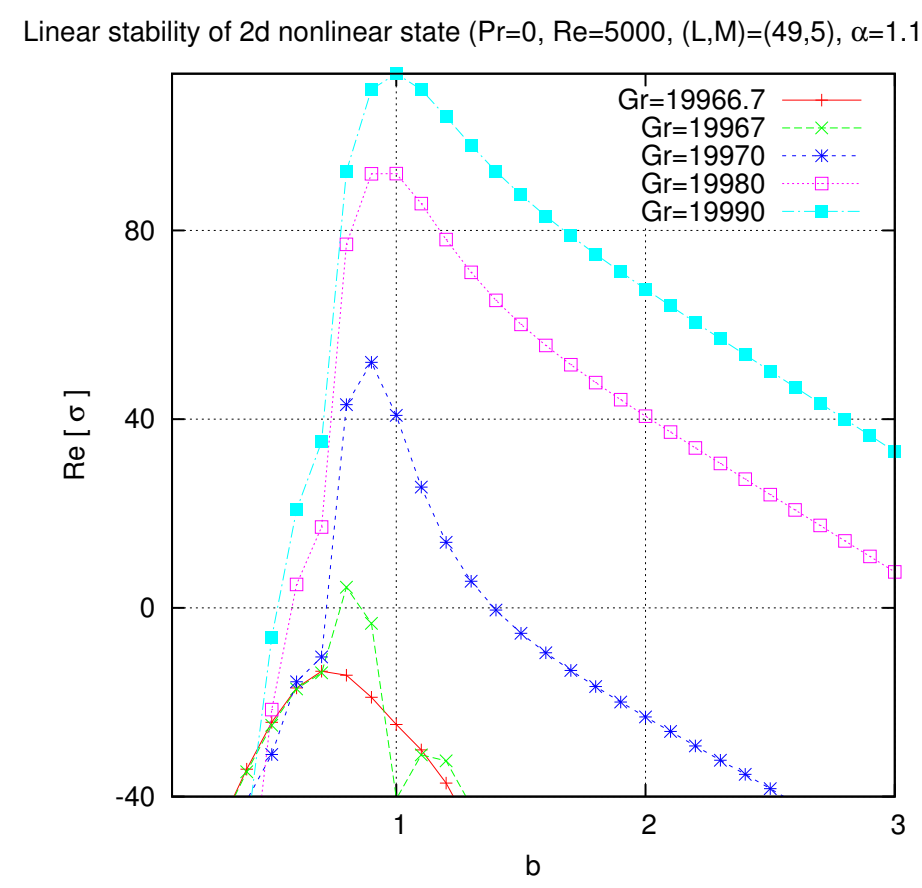

FIG. 9: Linear stability of two-dimensional nonlinear state for $\operatorname{Pr}=0$, $R e=5000, \alpha=1.12$, and $(L, M)=(49,5) . d=\alpha / 2$. Real part of most unstable eigenvalue is plotted with respect to Floquet parameter $b$.

cases with $b=0$ examined $\sigma_{r}$, the real part of $\sigma$ of equation (16), it was symmetric about, and attained its minimum value for $d=a / 2$. This property indicates that instabilities introduced in the form of vortices with twice the original wavelength are not preferred, a property already observed in previous non-linear studies of [2], [9] and [11]. We observe from Figure 8 that stable transverse waves in a vertical slot are those with a wave length close to the values of the critical wavelength, as predicted by [10] for values sufficiently close to $G r_{c}$ (of the neutral curve). There are of course discrepancies as the Grashof number increases. Similar conclusions, although not as pronounced, are also apparent for the $\operatorname{Pr}=0$ case, [12]. 
Oscillatory instabilities - subharmonic and superharmonic instability boundaries

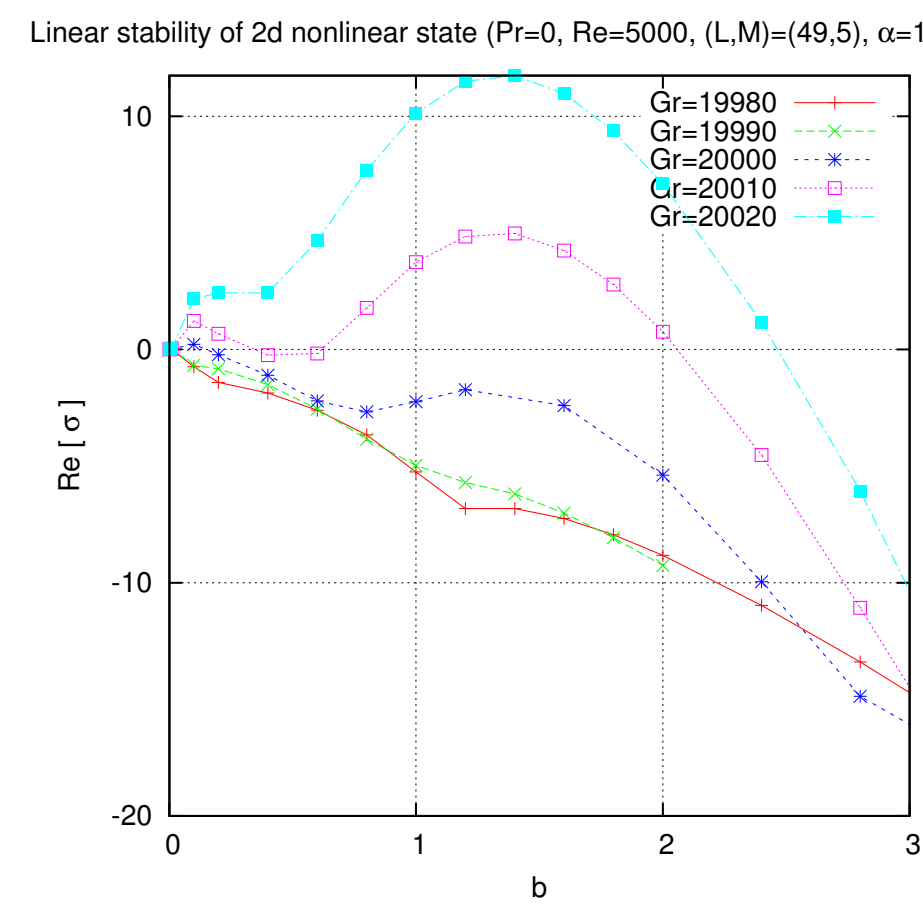

FIG. 10: Linear stability of two-dimensional nonlinear state for $\operatorname{Pr}=0$, $R e=5000, \alpha=1.12$, and $(L, M)=(49,5) . d=0$. Real part of most unstable eigenvalue is plotted with respect to Floquet parameter $b$.

The stability region is bounded from above by two bifurcation curves, both of which are determined by the change of sign on the real part of a single eigenvalue with vanishing imaginary part. The emerging three-dimensional flow is therefore phase-locked with the two-dimensional flow. For this section case $\sigma_{r}=\sigma_{r}(b, d)$ and in order to accurately determine the value of $G r$ at which $\sigma_{r}$ changes sign, maximum growth rates have been calculated for various values of $G r$ at a given wavenumber value. As before a positive $\sigma_{r}$ will indicate an instability for the particular state. For each value of $G r$ that characterizes a secondary solution, 
maximum $\sigma_{r}$ are first determined for fixed values of $b$ and then this value of $b$ is used to determine the optimum value of $d$, i.e. the value of $d$ for which the maximum $\sigma_{r}$ is observed. The value that determines the bifurcation curve for the corresponding three-dimensional flow, has been evaluated by interpolation. A large number of states were examined and we present the results of representative cases used for the determination of the subharmonic, Figure 9, and superharmonic, Figure 10, stability boundaries. In the same Figures we also depict the values of the Floquet parameters $(d, b)$ form which maximum growth rates were observed for each of the two cases. Since the subharmonic stability boundary occurs very close to the linear curve, we have depicted the superharmonic boundary in a different plot, so that both the subharmonic and super harmonic boundaries can be visualised.

\section{TERTIARY STATES}

The finding that two-dimensional vortex flow becomes unstable to threedimensional disturbances very soon after the Grashof number exceeds its critical value has stimulated the attempt to study three-dimensional solutions of the basic equations (NS of our paper). The computational cost is increased substantially in the step of elevating calculations from two to three dimensions, but we have adhered to the principle that the truncation level in three dimensions not only should be consistent with the truncation level of our studies, but should be increased until the difference in the coefficient values is minimised, by increasing $\{n, m, l\}$ of (equation number here). The results reported here for the tertiary solutions have employed the truncation level $\{N=M=5, L=45\}$. We describe below the properties of tertiary states that we identified. The contour plots of Figures 11 and 12 depict the fluctuating velocity field can be written in the form $\boldsymbol{u}=\boldsymbol{\nabla} \times \boldsymbol{k} \psi+\nabla \times \boldsymbol{i} \phi_{1}+\boldsymbol{\nabla} \times \boldsymbol{j} \phi_{2}$, where $\hat{\boldsymbol{i}}, \hat{\boldsymbol{j}}, \hat{\boldsymbol{k}}$ are the unit vectors in the streamwise, spanwise and wall normal, $x-, y-$ and $z$, directions respectively. Note 
that $\phi_{1}=\partial \phi / \partial y, \phi_{2}=-\partial \phi / \partial x$ are derivatives of the potential $\phi$ of equation (7) (see also [3]). This formula allows a three-dimensional graphic representation of the velocity field through contour plots of $\psi, \phi_{1}$ and $\phi_{2}$ in the planes perpendicular to $\hat{\boldsymbol{i}}, \hat{\boldsymbol{j}}, \hat{\boldsymbol{k}}$, as shown for typical examples of the superharmonic and subharmonic tertiary states in Figures 11 and 12.

\section{Symmetries of the superharmonic tertiary states}

The three-dimensional solutions bifurcate from the two-dimensional one through an instability that is superharmonic with respect to the $x$-dependence.

$$
\begin{aligned}
\phi= & \sum_{n=0}^{N} \sum_{\substack{m, l \\
(m, l) \neq(0,0)}}^{M, L}\left(1-z^{2}\right)^{2} T_{n}(z) \\
& \times\left[\hat{a}_{n m l} \cos (m \alpha(x-c t))+\check{a}_{n m l} \sin (m \alpha(x-c t))\right]\left\{\begin{array}{c}
\cos (l \beta y) \\
\sin (l \beta y)
\end{array}\right\}, \\
\psi= & \sum_{n=0}^{N} \sum_{\substack{m, l \\
(m, l) \neq(0,0)}}^{M, L}\left(1-z^{2}\right) T_{n}(z) \\
& \times\left[\hat{b}_{n m l} \cos (m \alpha(x-c t))+\check{b}_{n m l} \sin (m \alpha(x-c t))\right]\left\{\begin{array}{c}
\sin (l \beta y) \\
\cos (l \beta y)
\end{array}\right\},
\end{aligned}
$$

where $T_{n}(z)$ is the $n-t h$ Chebyschev polynomial and the weighted factors ensure the satisfaction of the boundary conditions. The tertiary solution of the present problem is characterized by several symmetry properties: The upper (lower) function must be chosen when $n$ is even (odd) for the case of $\phi$ and the upper (lower) function must be chosen when $l$ is odd (even) for the case of $\psi$. These symmetry properties include, of course, the two-dimensional secondary solution for which only coefficients with $l=0$ and even $m$ can be finite. The coefficients $\hat{a}_{n m l}\left(\check{a}_{n m l}\right), \hat{b}_{n m l}\left(\check{b}_{n m l}\right)$ vanish when $l$ is odd (even). As there is no temperature 


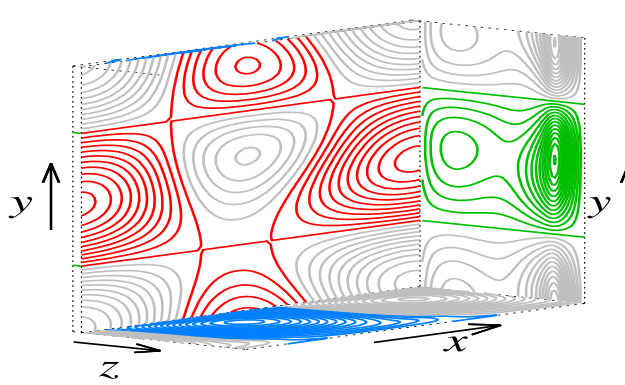

(a)

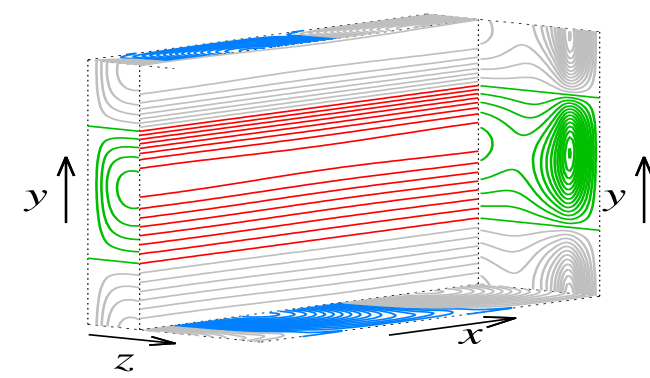

(c)

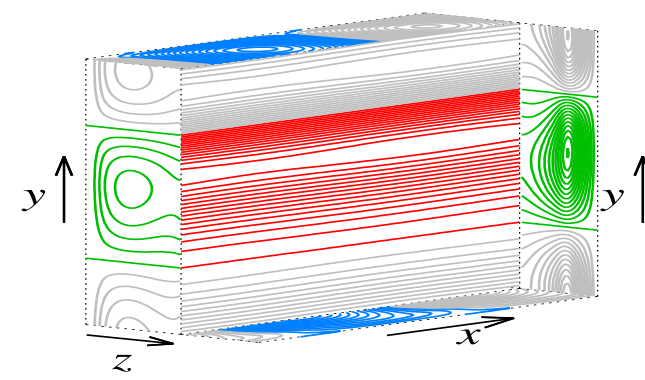

(e)

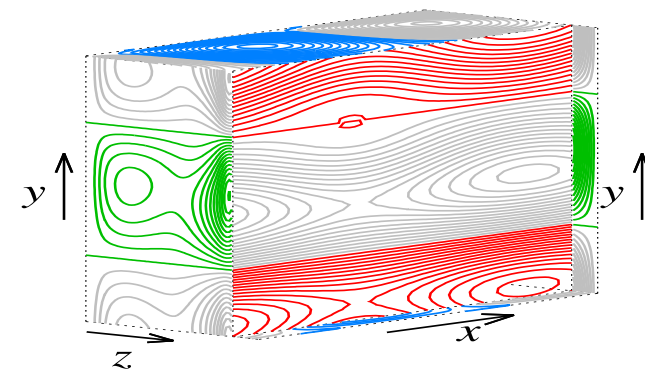

(g)

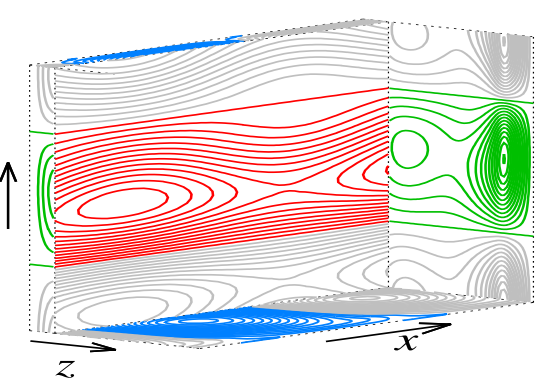

(b)

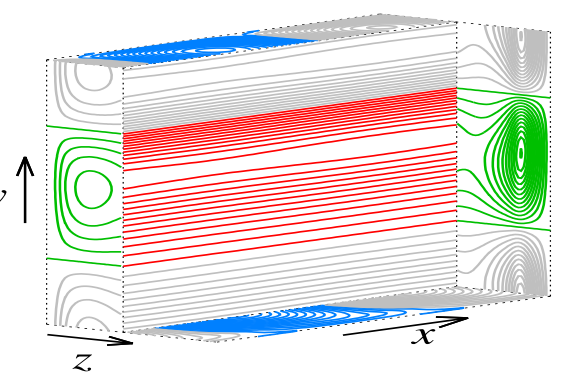

(d)

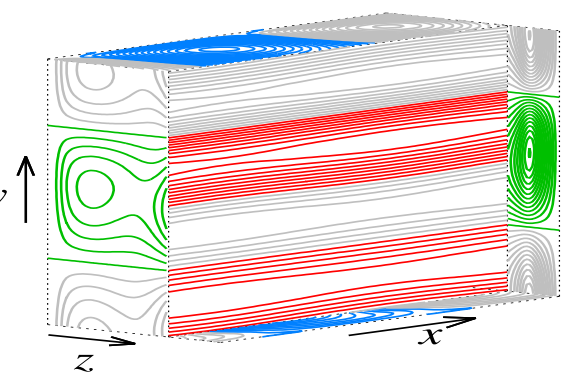

(f)

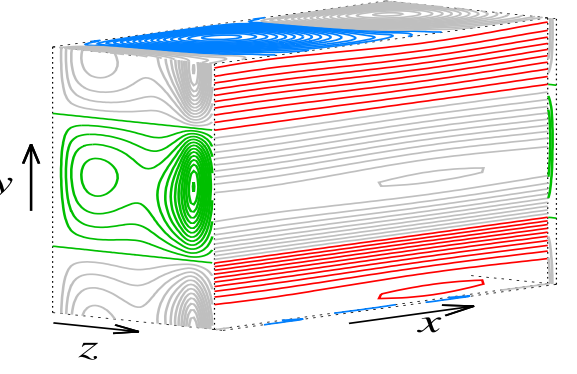

(h)

FIG. 11: (a)-(h): Contour plots for the superharmonic tertiary state for the Busse potentials (see [13]) for $z=-0.9,-0.7,-0.4,-0.1,0.1,0.4,0.7,0.9$. $(\operatorname{Pr}, G r, R e)=(0,20030,5000),(\alpha, \stackrel{2}{\beta})=(1.13,1.4),(L, M, N)=(49,5,5)$. 


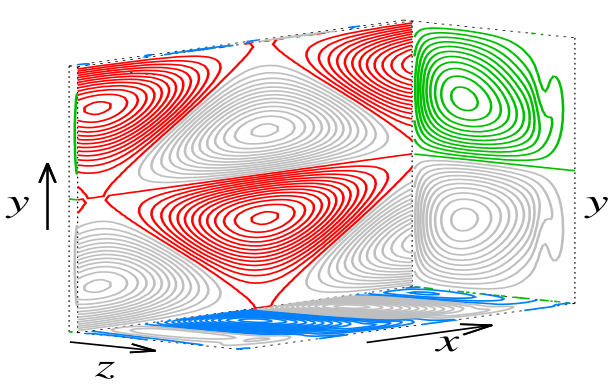

(a)

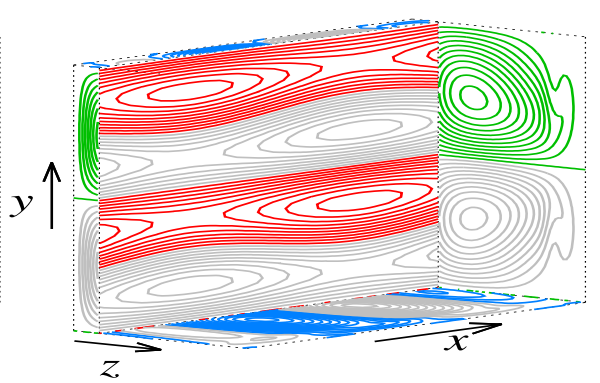

(b)

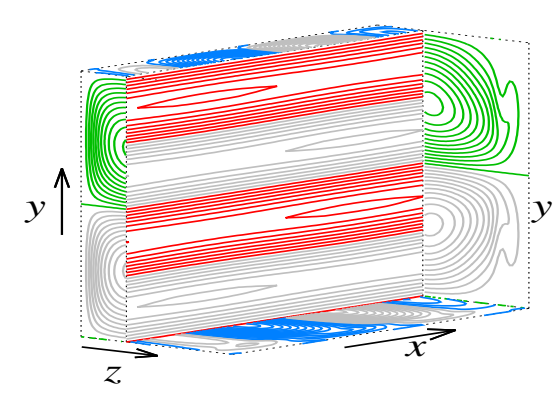

(c)

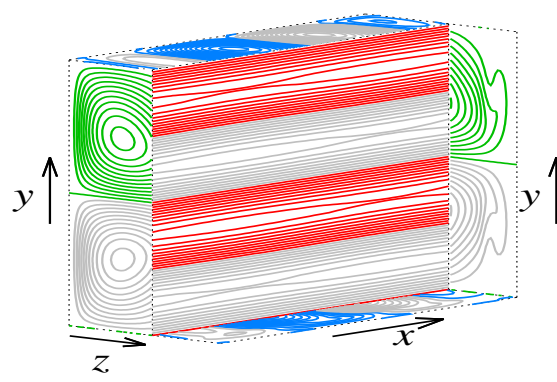

(e)

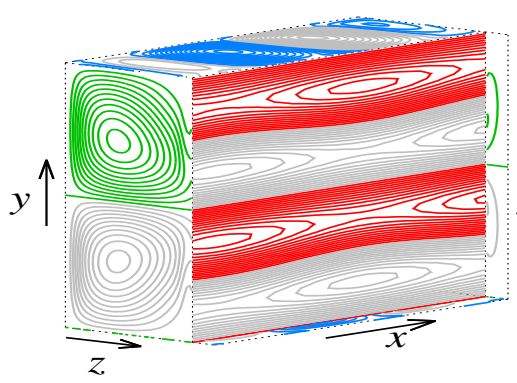

$(\mathrm{g})$

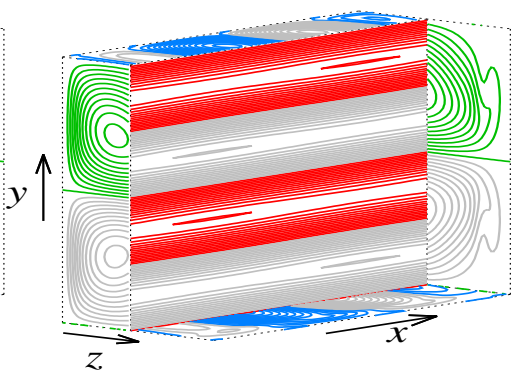

(d)

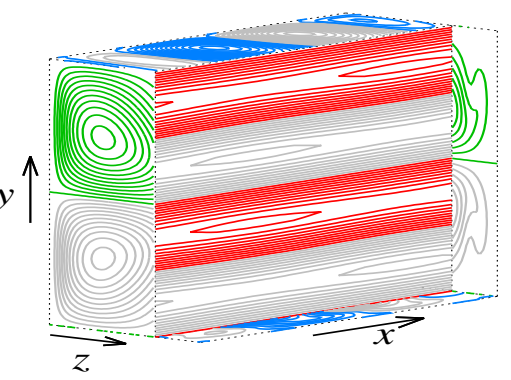

(f)

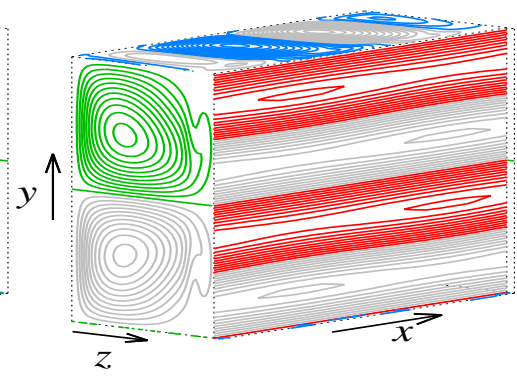

(h)

FIG. 12: (a)-(h): Contour plots for the subharmonic tertiary state for the Busse potentials (see [13]) for $z=-0.9,-0.7,-0.4,-0.1,0.1,0.4,0.7,0.9$. $(\operatorname{Pr}, G r, R e)=(0,20000,5000),(\alpha, \beta)=(0.56,0.85),(L, M, N)=(49,5,5)$. 
field involved in the calculations we do not discuss here the coefficients for $\theta$. The symmetries can be realised physically by half wave length translations in the stream- and span-wise directions, $\frac{\pi}{\alpha}$ and $\frac{\pi}{\beta}$ :

$$
\begin{aligned}
& \phi(\xi, y)+(-1)^{m} \phi\left(\xi+\frac{\pi}{\alpha}, y+\frac{\pi}{\beta}\right)=0 \text { for odd } l \\
& \phi(\xi, y)-(-1)^{m} \phi\left(\xi+\frac{\pi}{\alpha}, y+\frac{\pi}{\beta}\right)=0 \text { for even } l \\
& \psi(\xi, y)-(-1)^{m} \psi\left(\xi+\frac{\pi}{\alpha}, y+\frac{\pi}{\beta}\right)=0 \text { for odd } l, \\
& \psi(\xi, y)+(-1)^{m} \psi\left(\xi+\frac{\pi}{\alpha}, y+\frac{\pi}{\beta}\right)=0 \text { for even } l
\end{aligned}
$$

where we have introduced the coordinate $\xi=x-c t$ to both include the drifting super harmonic solutions with a phase velocity $c$, as well as simplify slightly the notation. Since the stability boundary from which the super harmonic solution bifurcates from is characterised by and eigenvalue whose imaginary part vanishes, the super harmonic solution is phase locked with the two-dimensional secondary solution.

\section{Symmetries of the subharmonic tertiary states}

The symmetries of the subharmonic tertiary solutions are well studied for the $\operatorname{Pr}=0$ case, see [2]. We mention them here again for completeness. Despite the introduction of the PFC component in the basic flow the coefficients of the subharmonic tertiary state satisfy:

$$
\begin{array}{cc}
m+l=\text { even } \& m+n=\text { odd }: & a_{n m-l}^{R}=a_{n m l}^{R}, \\
m+l=\text { even } \& m+n=\text { even }: & a_{n m-l}^{I}=a_{n m l}^{I}, \\
m+l=\text { even } \& m+n=\text { odd }: & b_{n m-l}^{I}=-b_{n m l}^{I}, \\
m+l=\text { even } \& m+n=\text { even }: & b_{n m-l}^{R}=-b_{n m l}^{R},
\end{array}
$$

where $a_{l m n}\left(=a_{l m n}^{R}+i a_{l m n}^{I}\right)$ and $b_{l m n}\left(=b_{l m n}^{R}+i b_{l m n}^{I}\right)$ are the complex coefficients of the poloidal and toroidal potentials of equation (equation number here) in the 
Fourier expansion:

$$
\begin{aligned}
\phi(x, y, z, t) & =\sum_{n=0}^{N} \sum_{\substack{m=-M \\
(m, l) \neq(0,0)}}^{M} \sum_{l=-L}^{L} a_{n m l}\left(1-z^{2}\right)^{2} T_{n}(z) \exp (i m \alpha \xi+i l \beta y) \\
\psi(x, z, t) & =\sum_{n=0}^{N} \sum_{\substack{m=-M \\
M}}^{L} \sum_{l=-L}^{L} b_{n m l}\left(1-z^{2}\right) T_{n}(z) \exp (i m \alpha \xi+i l \beta y) .
\end{aligned}
$$

All other coefficients, that are not explicitly mentioned in (22), vanish. This symmetry is the one that the HVS observes and its physical meaning can be found in [7]. In our case the subharmonic tertiary state is a drifting wave.

\section{CONCLUSIONS}

We have presented in this paper a detailed account of the secondary solutions, appropriate to incompressible LHF flow, and examined their stability. The main conclusion to be drawn from the investigation presented in this paper is that the secondary flow can be both supercritical and subcritical in its onset, depending on the values of the parameters $G r, \operatorname{Re}$ for $\operatorname{Pr}=0,0.71$ that we examined. The two-dimensional solutions do not penetrate completely the stable region as defined by the critical values (see Figure 2). This leaves a large area, for which the basic flow remains stable, even for relatively large values of $G r, R e$. Various aspects of the secondary flow have been presented with the general conclusion that $R e$ is more influential that the convective influence of $G r$. We have shown, for example, that the is mean flow and temperature distortions are greater for larger Re, rather than (equal) increase in $G r$. The ensuing numerical analysis of the stability of the (secondary) solutions has shown that the flow, when subjected to arbitrary three dimensional infinitesimal perturbations, undergoes both a subcritical, as well as a supercritical bifurcation, with the three-dimensional flow remaining phase locked with the two-dimensional flow on both cases. The subharmonic tertiary flow settles 
in very quickly, while the onset of the superharmonic flow is substantially delayed. There remains, of course, to identify the influence of the properties of the fluid on the flow. The results for higher values of $\operatorname{Pr}$ will be published elsewhere.

TA acknowledges the financial support from Marie Curie Sklodowska Programme of FP7 of the European Union. SG acknowledges financial support from ORDIST of Kansai University, where part of this work was conducted. TI acknowledges a Visiting Scholars fund from Aston University, where this work was completed.

* Corresponding author: s.c.generalis@aston.ac.uk

[1] N.I.Lobov, Perm. Translated from Izvestiya Akademii Nauk SSSR, Mekhanika Zhidkosti i Gaza 6, 130 (1978).

[2] M. Nagata and F. Busse, J. Fluid Mech. 135, 1 (1983).

[3] B. Schmitt and W. von Wahl, in Applications to the Boussinesq-Equations, in The Navier-Stokes Equations II, Theory and Numerical Methods, Vol. II, edited by R. R. J. G. Heywood, K. Masuda and S. A. Solonnikov (Springer Lecture Notes in Mathematics, 1992) pp. 291-305.

[4] T. Herbert, AIAA Paper 18, 1125R (1980).

[5] T. Akinaga, T. Itano, and S. Generalis, Arxiv (submitted to Eur. Phys. Lett.). (2015).

[6] S. Generalis and K. Fujimura, J. Phys. Soc. Japan 78, 084401 (2009).

[7] T. Itano and S. Generalis, Phys. Rev. Lett. 102, 4 (2009).

[8] S. Generalis and T. Itano, Phys. Rev. E 82 (2010).

[9] A.Chait and S.A.Korpela, J. Fluid Mech. 200, 189 (1989).

[10] W. Eckhaus, Studies in Non-Linear Stability Theory (Springer-Verlag, 1965).

[11] S. Generalis and M. Nagata, J. Heat Transf.-Trans. of ASME 125, 795 (2003). 
[12] M.Nagata, Eur. J. Mech. B/Fluids 17, 33 (1998).

[13] E. Weisshaar, F. H. Busse, and M. Nagata, J. Fluid Mech. 226, 549 (1991). 